Recherches en didactique des langues et des cultures

Les cahiers de l'Acedle

14-1 | 2017

Notions en questions - Emergentisme

\title{
Réponse à Marjolijn Verspoor : de la nécessité d'aller au-delà du linguistique
}

Jean-Paul Narcy-Combes

\section{OpenEdition}

Journals

Édition électronique

URL : http://journals.openedition.org/rdlc/1141

DOI : $10.4000 /$ rdlc. 1141

ISSN : 1958-5772

Éditeur

ACEDLE

Référence électronique

Jean-Paul Narcy-Combes, «Réponse à Marjolijn Verspoor : de la nécessité d'aller au-delà du

linguistique », Recherches en didactique des langues et des cultures [En ligne], 14-1 | 2017, mis en ligne le 23 décembre 2016, consulté le 01 mai 2019. URL : http://journals.openedition.org/rdlc/1141 ; DOI

$10.4000 /$ rdlc. 1141

Ce document a été généré automatiquement le 1 mai 2019.

\section{c) (i) $९$}

Recherches en didactique des langues et des cultures is licensed under a Creative Commons AttributionNonCommercial-NoDerivatives 4.0 International License 


\title{
Réponse à Marjolijn Verspoor : de la nécessité d'aller au-delà du linguistique
}

\author{
Jean-Paul Narcy-Combes
}

\section{Introduction}

1 Le format des journées NEQ conduit des chercheurs de domaines voisins, et toutefois différents, à dialoguer pour explorer une notion, ici l'émergentisme, et à apporter leur éclairage en réponse à un positionnement donné. Le faire est une entreprise délicate, elle conduit le répondant à se positionner clairement, à déconstruire et discuter la communication originale afin de mettre en valeur ses points forts et d'émettre les réserves ressenties. Un positionnement épistémologique précédera celui plus directement concerné par le domaine, avant d'aborder la communication de Marjolijn Verspoor (MV dorénavant) elle-même. Le débat sera abordé sous le prisme de la didactique de l'intervention (Narcy-Combes, 2005 ; Bertin et al., 2010), cadre dans lequel se situe le répondant.

2 Sans doute autant une réponse aux débats de la journée qu'un besoin suscité par l'article de MV, ce positionnement se fera selon deux axes, la transdiciplinarité d'une part, puis le construit d'émergence. Enfin, sera décrite la posture scientifique qui conditionne le regard porté sur le texte de MV.

\section{Transdisciplinarité}

3 Pour Claverie (2010), l'idée d'une unité scientifique a été impulsée quand la monodisciplinarité positiviste n'a plus suffi à répondre à la complexité des questions qui se posaient. En réponse à cette complexité, la posture transdisciplinaire cherche, au-delà des disciplines, une vision de l'humain dans sa globalité et inscrit dans le monde (Claverie, 2010: 20). Cette posture entre en résonance avec mes questionnements de 
chercheur (Narcy-Combes, 2014) et avec les interrogations d'autres chercheurs de notre domaine : ainsi, un article récent, publié par le Douglas Fir Group (2016), propose une synthèse des interrogations scientifiques en linguistique appliquée qui peuvent trouver des réponses dans une appréhension transversale des disciplines et la définition qu'on y trouve de la transversalité correspond à celle de Claverie. Une proposition y est faite de déterminer des activités thématiques :

Rather than privileging the disciplines « as the locus of intellectual activity, while building bridges between them, or assembling them into a collection, » Halliday advocates creating "new forms of activity which are thematic rather than disciplinary in their orientation » (Douglas Fir Group, 2016: 24) $)^{1}$.

Ce construit d'activité thématique semble correspondre aux réflexions autour de l'émergentisme auxquelles la journée NEQ de janvier 2016 et ce volume sont consacrés. Une telle perspective conduit à privilégier les méthodologies de recherche mixtes (mixed methods research, voir Dörnyei, 2007) où les outils (mesure, étude de cas, etc.) ne conduisent pas à des preuves, mais à des pistes de réflexion qui nécessitent que le chercheur explicite son positionnement. Nous ne pouvons que rejoindre le collectif d'auteurs de l'article (Douglas Fir group, 2016) quand ils mettent en avant le rôle critique et transgressif de la transdisciplinarité, comme le montrera la conclusion de cet article.

\section{L'émergen-ce/tisme}

5 Le terme contient de toute évidence une métaphore, ce qui n'est pas sans influencer la réception qui en est faite. Parmi les définitions disponibles d'émerger, celles que propose pour l'anglais Webster (en ligne) correspond à un positionnement émergentiste : « to rise or appear from a hidden or unknown place or condition, to come out into view, to become manifest ».

6 Elle est complétée de façon cohérente par la Stanford Encyclopedia of Philosophy (O'Connor et al., 2015, en ligne)

Emergent entities (properties or substances) 'arise' out of more fundamental entities and yet are 'novel' or 'irreducible' with respect to them. (For example, it is sometimes said that consciousness is an emergent property of the brain) ${ }^{2}$.

7 En français, la métaphore de l'émergence n'échappe pas aux effets que Bachelard signale (1936 [1970]: 78). Le discours/la parole sont de nature radicalement différente des processus qui contribuent à leur production/réception. Il y a donc rupture de nature entre les uns et les autres, et rapprocher l'émergence dans notre acception et l'émergence d'une partie d'un iceberg, comme cela a été fait lors des débats de la journée $\mathrm{NEQ}^{3}$, n'est pas vraiment opératoire. Pour l'iceberg, la partie immergée est de même nature que la partie émergée et les conditions de l'émergence n'ont rien à voir avec l'iceberg lui-même, mais avec des phénomènes de densité.

\section{Posture scientifique du chercheur ${ }^{4}$ en ce qui concerne la cognition}

8 Le positionnement, exprimé ici, a été très influencé par LeDoux (2003 : 38). Pour lui, la neurophysiologie pourrait relever d'une réflexion nomothétique (les humains sont très semblables sur ce plan), mais la psychologie relèverait de l'idiographique (Ibid.) car chaque individu a son histoire dans des contextes variables, ce qui façonne les mécanismes neurophysiologiques de manière différente. La part que la neurophysiologie 
donne aux émotions conduit à relativiser la rationalité de la réflexion comme le fait Damasio (1995). Disons, de manière très synthétique (Narcy-Combes, 2005), qu'une situation sociale donnée - c'est-à-dire la confrontation à la "réalité » extérieure à l'individu - va le conduire à réagir en fonction d'une émotion, pour lui spécifique à ce type de situation et à ce qu'il doit y accomplir, qui se combine aux émotions du moment. Il agit alors en fonction de schèmes de pensée et de comportements déjà en place ou de routines d'action(s) (Piaget, 1970; Claverie, 2010). Ces phénomènes existent tant au niveau d'actions routinières, qu'à celui d'actions socialement et cognitivement plus complexes. Les individus accèdent à leurs attitudes, à leurs émotions et autres états intérieurs, par l'observation de leurs comportements ou les réactions d'autrui, car les indices internes ne sont pas facilement perceptibles et interprétables (Channouf, 2004). On parle d'inconscient cognitif qui conduit les actions sans que les sujets soient toujours conscients de ce qui les détermine, même dans des situations très complexes telles que la pratique pédagogique; on parle alors de compétences incorporées à l'action (Lenoir, 2007). Le conditionnement est modifiable, non sans un nouvel entraînement qui va audelà du savoir (Narcy-Combes, 2005), le fait de savoir ce qu'il est préférable de faire ne suffit pas toujours à modifier les comportements. L'obstacle majeur à un tel changement est qu'en fait, le maintien de tout comportement conditionné protège de toute déstabilisation.

Les recherches sur le mode de fonctionnement de la pensée et du comportement des humains soulignent que l'analogie serait la base essentielle de ce fonctionnement, même si cette analogie génère des comportements cognitifs et sociaux très différents suivant les cultures. A coup sûr, l'analogie se reflète dans la métaphore, dont l'emploi est constant dans les descriptions scientifiques (Hofstadter et Sander, 2013), et, dans nos domaines, elle explique comment ont été déterminés les construits d'assimilation/nativisation (Piaget, 1970, Andersen 1983), et le fait que les prédictions/interprétations se font par projections de nature analogique (Gigerenzer, 2009) qui conduisent à des réactions conditionnées qui seront rationalisées. Dans ce cadre, on comprend que le savoir soit vu comme le résultat d'une co-construction située constamment remise en cause (Lave \& Wenger, 1991) et que la connaissance soit distribuée (Hutchins, 1995), entre autres, parce que l'interaction sociale déclenche les processus et amène à questionner les constructions individuelles.

De ce fait, comme l'a rappelé Loewy dans son intervention à la journée NEQ : Every act in every moment is the emergent product of context and history, and no component has causal priority", et "using is learning and doing is learning " ce qui nous renvoie à Freinet (1964) d'une part, et à Piaget (1970), d'autre part, et souligne que tout apprentissage est interactif.

Les caractéristiques et les relations humaines ont initialement des fondements d'ordre biologique. Le biologique est universel, mais ce qui émerge est individuel. La métaphore de l'ordinateur ne saurait convenir à l'humain, car les réseaux cognitifs sont à la fois le soft et le hard. Un retour sur l'évolution du langage chez l'humain, depuis les débuts d'un probable protolangage, pour lequel nous nous appuierons sur Miras (2014), nous conduit à relativiser notre positionnement lié à Simondon (1989). Certes il existe une relation transductive entre humain, technologie et société (voir Narcy-Combes, 2005), mais la transformation n'est pas due qu'aux seules pressions sociales sur des facteurs physiologiques, des changements dans l'environnement ${ }^{5} \mathrm{y}$ auraient également contribué et favorisé l'apparition (l'émergence) d'une capacité langagière complexe dans une 
perspective évolutionniste. Il reste à comprendre si la conscience était antérieure au langage, ou si elle a « émergé » des échanges que celui-ci permettait.

Selon Vygotski (1997 [1934]), la pensée n'est pas seulement exprimée par les mots : elle vient à l'existence à travers les mots. La neuro-imagerie ne permet d'observer que des processus neuro-cognitifs, pas ce qui en émerge, ce qu'on appelle la pensée, qui est inféré et reconstruit à partir du discours, des comportements et dans les réalisations comportementales, artistiques, scientifiques qui sont observables. Ce que les instruments révèlent dans le cerveau, ce sont des corrélats biologiques (activité électrique, variation du flux sanguin) de ce qui se passe quand un être humain "pense ». De plus, une même pensée peut être le produit d'états cérébraux différents, sensible à la variété des histoires individuelle (Guillaume et al., 2013), la pensée ne peut donc être comprise, décrite, expliquée en dehors de son contexte individuel, culturel et social et elle le sera, si on peut dire, à postériori. Laplane (2001 : 345) écrit :

La thèse ici défendue est qu'une pensée ne peut être complète sans l'intervention du langage, mais qu'elle existe largement préformée sur un mode non verbal et que le langage participe, de ce fait, à son parachèvement.

L'activité mentale se structure d'une façon qui ne correspond pas à l'organisation du discours, c'est pourquoi je serais tenté de postuler que ce que nous appelons la pensée est une reconstruction du jeu des sensations/perceptions/émotions sur les réactions qui ne deviennent cognitives que lorsque le discours intérieur ou extérieur lui a donné une «forme » accessible qu'il importe parfois de déconstruire. Les organisations symboliques externes ne correspondent pas au fonctionnement interne des réseaux dits cognitifs, ce qui pose un problème didactique majeur, car les conceptions traditionnelles décrivent l'organisation interne comme si elle correspondait à ce que révèle le discours. Il semble, de plus, impossible de dissocier discours/pensée/contenu, ce qui n'est pas sans conséquence pour l'apprentissage des « langues ».

Il est possible alors d'avancer que ce qui «émerge » sont des pratiques qu'on ne saurait qualifier simplement de langagières, en particulier du point de vue de leur production. Elles se réalisent et sont observables sous la forme de comportements, relevant d'un système émotionnel, qui conduit à des informations et à de la communication certes, mais aussi à la construction et à la perception de soi, avec une variabilité qui mérite d'être prise en compte et qui dépend du contexte, de l'histoire, etc.

15 Ainsi les réflexions du Douglas Fir Group (2016) permettent de penser que l'on pourrait rejeter les orientations épistémologiques rationalistes et pragmatistes et proposer des dispositifs d'apprentissage qui prennent en compte le fait que discours, contenuS et sociétés/cultureS sont indissociables, et qui, donc, aillent réellement au-delà d'un travail axé sur la «langue». Il importerait également qu'ils soient co-construits avec les participants, en s'adaptant constamment à ce que les circonstances de l'apprentissage révèlent au lieu de suivre un programme pré-établi (construit d'organizing circumstances, Spear and Mocker, 1984), etc. Ce positionnement va de pair avec un engagement politique et éthique (Narcy-Combes 2005 ; Corijn, 2006 ; Beacco 2013) puisque l'interaction nous met en contact avec les problèmes de la cité.

\section{Posture scientifique du chercheur : didactique des langues}

Ce positionnement est naturellement intimement lié à ce qui précède. Je suis convaincu qu'il convient de distinguer langue et discours/parole. Ce qu'on appelle les « langues » est 
lié à des histoires et à des projets qui ont conduit à l'instauration des sociétés. Le construit de langue relève souvent de l'idéologie. Les langues sont alors supposées stables, universelles, voire " pures » (cf. le rôle de l'Académie française), mais le discours/ parole est, lui, fondamentalement instable, contextualisé, historicisé, variable. Si la différence est rarement prise en compte dans le quotidien et la vie sociale, il conviendrait qu'elle le reste dans nos réflexions. Les «langues", certes, remplissent un rôle fonctionnel, instrumental et communicatif au niveau abstrait, mais le discours/parole résulte/permet des expériences, principalement identitaires et relationnelles (Kramsch, 2002).

Apprendre une ou des « langues étrangères ", revient alors à confronter des perceptions / sensations / interprétations / traductions du monde, en fonctions d'histoires, d'entreprises, d'intentions ou de visées potentiellement différentes, voire conflictuelles. Il ne s'agit plus du tout de faire fonctionner des mécanismes (qui seraient identiques quelles que soient les langues), ni de produire des comportements adaptés, mais de permettre de vivre de nouvelles expériences afin d'augmenter son bagage relationnel et humain en fonction d'objectifs de préférences personnels, mais souvent institutionnels: le mécanisme n'est en fait pas linguistique, mais neurophysiologique, conditionné par des facteurs socio-émotionnels, socioculturels, sociopolitique et idéologiques (Douglas Fir Group: 2016). Par contre, le résultat, le discours, est souvent décrit en termes linguistiques. Ce qui précède justifie la conception de dispositifs/situations d'apprentissage avec un enjeu social et relationnel et la prise en compte de LangueS / contenuS / cultures et des contextes et institutions où nous intervenons. Cette complexité disciplinaire, qui va bien au-delà du champ de la didactique des langues et des sciences du langage ${ }^{6}$, nous ramène à la transdisciplinarité.

En Recherche en Acquisition des Langues (RAL), les chercheurs s'intéressent aux aspects universels de l'acquisition du langage, mais les variations sont conséquentes puisqu'audelà de l'universalité des processus, la vie des individus les rend très différents, même dans des contextes proches. L'apprentissage d'une langue ne consiste pas à mettre ensemble des éléments prédéterminés comme on l'a souvent cru (Narcy-Combes, 2005). Il convient d'établir des liens complexes entre histoire, contexte, perception, intention (alité), action, réaction, en essayant de stabiliser ce qui en résulte par l'action sociale. Il n'y a pas de mécanismes spécifiques d'apprentissage, mais des réseaux qui se mettent en place en fonction d'une logique dont la complexité nous échappe partiellement. On est loin d'une "pensée technique» (Heidegger, 1973), notre rationalité est limitée et n'échappe ni à notre histoire ni à nos émotions.

En ce qui concerne notre domaine, rappelons qu'il parait difficile d'isoler discours, contenuS et cultureS/social. Le $" \mathrm{~S}$ » de contenuS et de cultureS tente de prendre en compte la multiplicité des éléments dans l'expérience d'un individu pluriel (Lahire, 1998).

Cette réflexion préalable justifie les conceptions théoriques plus directement liées à l'apprentissage des langues qui vont suivre. Il est possible de postuler la complémentarité des théories émergentiste (O'Grady, 2010; Ellis, 1998) et socioconstructiviste (Vygotski, 1997 [1934]), en suivant des auteurs tels que De Bot et al. (2013) qui soulignent les apports de cette complémentarité. Suivant les parcours, il semblait important de conserver le besoin d'amorcer un processus de dénativisation/accommodation (Andersen 1983; Piaget 1970, Grosbois 2011) face aux processus de traitement des «langues» déjà connues (des codes en place) et face aux phénomènes complexes d'activation plurielle de ces codes en fonction des situations et des problèmes. Jessner (2006) les décrit en nous rappelant 
que les individus plurilingues représentent «la» norme puisque les individus monolingues restent minoritaires. Ce positionnement théorique combine ainsi une perspective socioconstructiviste du langage (Vygotski, 1997 [1934]) selon laquelle le développement est déclenché principalement par l'interpsychique, et prend en compte le fait que chaque individu construit sa propre connaissance et en affine la gestion (intrapsychique). Les difficultés rencontrées par les apprenants lors de l'apprentissage leur sont spécifiques dans ce cadre.

Un changement qualitatif du système psycholinguistique au fur et à mesure qu'un individu avance dans l'acquisition d'une ou de plusieurs langues est perceptible (Jessner, 2006), et une compétence particulière se développe chez les plurilingues qui va au-delà de la simple mise en parallèle d'une nouvelle compétence de même ordre (Cummins, 1994). Différents chercheurs (citons Cenoz \& Gorter, 2011; Herdina \& Jessner, 2006) avancent que l'acquisition plus ou moins avancée de plus de deux langues déclenche une réorientation cognitive qui facilite l'acquisition de nouvelles langues. Monolingues, bilingues et plurilingues diffèrent donc qualitativement sur le plan du développement langagier. Les influences interlinguales, selon Cenoz \& Gorter (2011), favorisent des transferts conceptuels d'une langue à l'autre, de la L1, mais aussi des autres langues apprises, et varient selon la proximité des nouvelles langues apprises avec la L1 ou les autres langues et le degré de maîtrise des différentes langues. Certains chercheurs vont jusqu'à décrire ce qu'ils appellent "translanguaging », défini par Williams \& Hammarberg (1998) comme " the ability of multilingual speakers to shuttle between languages, treating the diverse languages that form their repertoire as an integrated system $»$. Ce concept décrit la capacité qu'ont les individus plurilingues de faire appel aux différentes ressources de leur répertoire langagier, avec une intentionnalité plus ou moins consciente, pour participer aux échanges de manière efficace en fonction des contextes et des situations.

Comment ainsi ne pas se retrouver dans la citation suivante :

Further, when it comes to explaining what learning is, at least conceptually and often empirically, our various theories stipulate the mutual entailment of the cognitive, the social, and the emotional and not the cognitive alone (The Douglas Fir group 2016: 21).

23 Il nous reste à répondre à notre collègue, en évaluant dans quelle mesure son positionnement et les expériences qu'elle décrit se situent dans le cadre qui vient d'être exposé.

\section{Regard sur l'article de Marjolijn Verspoor}

La réflexion qui est développée dans l'article est bien annoncée dans le titre, The development of linguistic complexity in second language development: A complex dynamic perspective, et le positionnement est clairement explicité :

As shown by a growing body of research, language is a dynamic system and language development is a dynamic process characterized by variability (intraindividual changes) and variation (inter-individual differences). This implies that the development of linguistic complexity is an individually owned process and we should not assume a priori that generalization beyond the individual is possible (MV). initiale que la complexité linguistique est un descripteur valide de la performance en L2 
comme indicateur de développement et de progrès confirme que l'approche reste dans le cadre des sciences du langage dites « dures » (linguistique et psycholinguistique).

\section{idiographique (cf. LeDoux, 2003) :}

1. Our advanced learners are similar in their linguistic complexity development in broad measures,

2. Our advanced learners are not similar in their linguistic complexity development in specific measures,

3. Our advanced learners have individually owned trajectories in developing linguistic complexity.

\section{Résultats}

Il est intéressant de lire que la description de la progression est générale :

At the lower end differences between levels were found in specific measures in the lexicon, in the middle levels the growth was found especially in specific syntactic measures, and at the end differences were found in specific lexical measures again (MV).

Ces résultats confirment que les différents sous-systèmes évoluent selon des étapes différentes, ce qui rappelle des travaux antérieurs des auteurs et d'anciens travaux, dans une autre perspective, rapportés dans Narcy (1990).

Il est intéressant de noter que les choix et l'attention des apprenants concernant certains aspects linguistiques entraînent des régressions dans d'autres points. Ceci rappelle les résultats obtenus par Khreim (2008) : l'attention portée à la prononciation, et la progression qui en résultait avaient conduit à une régression relative de la correction grammaticale chez des apprenants arabophones de l'anglais. 


\section{la complexité avec le temps, les études de cas sont plus pertinentes que des études de} groupe. Il serait intéressant de déterminer leur complémentarité.

\section{Réflexions et questions en fonction du positionnement}

Dans mes conceptions (voir plus haut), l'écrit, surtout académique, est une forme de discours d'autant plus proche de la « langue » que, lors de sa réception et production, la pression temporelle et sociale est réduite et que le scripteur a tout loisir de se référer à des ressources pour s'aider. 
Si MV peut écrire que :

For English as an L2 writing teachers, this study has shown that even students who have an L1 that is quite similar to the L2 in many respects and who are quite advanced when they enter university, still need at least three years of a great amount of input and practice to develop a linguistic style that can be considered academic (as indicated by the expert ratings),

il n'en reste pas moins vrai que le contexte et ses effets sont peu explicités et qu'il n'y a aucune mesure du contenu (en unités/grains de contenus par exemple), ou de l'adéquation pragmatique des textes. Les expériences que nous avons menées et les résultats d'autres recherches (Bozhinova et al., 2016) montrent qu'il s'agit d'un développement où une évolution est délicate à mettre en place. Le prendre en compte est nécessaire d'autant que les résultats de MV indiquent une influence de la tâche, ou du contenu, sur ce développement. Dans nos conceptions (Narcy-Combes, 2005), le contenu prime sur la «langue », ne mesurer que la complexité de celle-ci et son développement donne alors des résultats qui restent à compléter.

41 Il parait utile de savoir comment l'écrit a été construit, en particulier s'il y a eu des pratiques ouvertement translangagières et/ou métalinguistiques, utilisant toutes les ressources TIC disponibles, et de la collaboration. Des recherches (Bozhinova et al., 2016) où de telles pratiques ont été mises en place et observées donnent des résultats positifs, mais il est nécessaire d'obtenir des données longitudinales pour en mesurer les effets sur le long terme. Les effets des pratiques métalinguistiques et de leur poids cognitif ont été mis en relief par Roehr-Braken (2014) et nous n'y avons pas accès ici.

Il serait utile de disposer de données qui révèlent les effets des méthodologies d'enseignement et de la culture éducative et qui permettent de comparer avec un apprentissage informel. Enfin, il resterait à déterminer comment reproduire l'expérience afin de mesurer les influences des langues et du plurilinguisme, du contexte et des individus, sachant que même dans les deux cas étudiés peu de données sont disponibles sur ces points. On peut se demander si les méthodologies employées permettraient de mesurer l'influence des paramètres personnels, sociaux et culturels, et finalement si une approche réellement transdisciplinaire est possible au-delà d'un effet de programme comme le propose le Douglas Fir group.

\section{Discussion conclusive}

43 Ce qui intéresse MV, ce n'est pas uniquement ce qui émerge, elle mesure le développement qui fait suite à un travail dont les modalités ne sont pas connues parce qu'elle n'opère pas dans l'intervention et que son domaine de recherche s'intéresse aux résultats. Dans son intervention en janvier, dont le contenu différait sensiblement du texte actuel, en montrant des pratiques d'enseignement, MV a souligné qu'elle était sensible à ce qui se passe pour chacun des apprenants. Même avec ces restrictions, cette recherche se situe dans la complexité, par contre, la transdisciplinarité au sens où je la verrai en intervention reste limitée.

En ce qui concerne les recherches plus approfondies que MV suggère, l'intervention nécessiterait d'aller plus loin. Les effets des différentes langues de l'apprenant sont importants, mais tant d'autres paramètres le sont également. Par contre, aucun chercheur ne serait en mesure de mener des recherches sur tous les fronts et nous ne pouvons qu'apprécier les résultats d'études telles que celles que MV nous rapporte: la

Recherches en didactique des langues et des cultures, 14-1 | 2017 
transdisciplinarité requiert un travail d'équipe et d'échange multidisciplinaire comme le propose le Douglas Fir Group (2016). MV nous rappelle que nous devons reconnaître les limites des mesures de groupe en nous sensibilisant aux variations individuelles, en conséquence nous devrions reconsidérer les pratiques et les critères d'évaluation qui règnent depuis plus de dix ans en référence au Cadre européen commun de références pour les langues (CECRL) d'une façon qui risque parfois d'être doxique (Castellotti et Nishiyama, 2011) et où on ne questionne plus la validité ontologique des niveaux (NarcyCombes, 2010). Notre rôle se situe également dans la remise en cause des pratiques institutionnelles, comme il a été écrit plus haut, si les recherches les interpellent.

En ce qui concerne la transdisciplinarité, l'intervention et notre posture émergentiste nous conduisent à prendre un positionnement qui va au-delà des sciences du langage ou de la linguistique appliquée, alors que l'article de MV s'y arrête. Aller plus loin ne lui aurait pas permis d'atteindre la même exhaustivité. C'est bien la carte de la complémentarité et du dialogue qu'il convient de jouer, ce qui m'amène à espérer en conclusion qu'un Douglas Fir Group européen verra le jour, avec sans doute des prises de position différentes de celles de nos collègues nord-américains.

\section{BIBLIOGRAPHIE}

Andersen, R. (1983). Pidginisation and Creolisation as Language Acquisition. Rowley, Newbury House.

Bachelard, G. (1936 [1970]). La Formation de l'esprit scientifique. Paris : Vrin.

Beacco, J. C., ed. (2013). Ethique et politique en didactique des langues - autour de la notion de responsabilité. Paris : Didier.

Bertin, J-C., Grave, P. \& Narcy-Combes, J-P. (2010). Second Language Distance Learning. Theoretical Perspectives and Didactic Ergonomics. Hershley, PA: IGI Global.

Bozhinova, K., Narcy-Combes, J-P. \& Zaouali, S. (à paraître, décembre 2016). « La production écrite vue comme un processus bilingue : dans quelle mesure les TIC peuvent-elles aider?». Pratiques, le Déjà là, n . 169-170.

Castellotti, V. et Nishiyama J. (dir.) (2011). « Contextualisations du CECR. Le cas de l'Asie du SudEst » Le français dans le monde. Recherche et applications, $n^{\circ}$ 50. Paris : CLE International-FIPF.

Cenoz, J. \& Gorter, D. (eds.) (2011). « A Holistic Approach in Multilingual Education: Introduction. Special issue Toward a Multilingual Approach in the Study of Multilingualism in School Contexts ». The Modern Language Journal. 95 : 3.

Channouf, A. (2004). Les influences inconscientes. Paris : Armand Colin.

Claverie, B (2010). « Pluri-, inter-, transdisciplinarité : ou le réel décomposé en réseaux de savoir ». Projectics / Proyéctica / Projectique 2010/1 (nº 4). pp. 5-27.

Cummins, J. (1994). « Knowledge, power, and identity in teaching English as a second language ». In Genesee, F. Educating second language children: The whole child, the whole curriculum, the whole community. Cambridge : Cambridge University Press. pp. 33-58. 
Corijn, E. (2006). Eléments d'un projet pour Ixelles. Une contribution citoyenne. Bruxelles : Parcours Citoyen.

Damasio, A. R. (1995). L'erreur de Descartes, la raison des émotions. Paris: Odile Jacob.

de Bot, K., Lowie, W., Thorne, S. L., \& Verspoor, M. (2013). « Dynamic Systems Theory as a Theory of Second Language Development ». In Mayo, M, Gutierrez-Mangado, M. \& Adriàn, M. (eds.). Contemporary Approaches to Second Language Acquisition. Amsterdam: Jon Bejamins, pp. 199-220.

Dörnyei, Z. (2007) Research methods in applied linguistics: Quantitative, qualitative and mixed methodologies. Oxford: Oxford University Press.

The Douglas Fir Group (2016). « A Transdisciplinary Framework for SLA in a Multilingual World ». The Modern Language Journal, 100 (Supplement 2016) DOI: 10.1111/modl.12301 0026-7902/16/1947.

Ellis, N.C. (1998). « Emergentism, connectionism and language learning ». Language Learning, 48 : 4, December. pp. 631-664.

Freinet, C. (1964). « Les techniques Freinet de l'École moderne ». Carnets de pédagogie pratique $\mathrm{n}$ -326. Paris : Armand Colin.

Grosbois, M. (2011). « CMC-based projects and L2 Learning: confirming the importance of nativisation ». ReCALL, vol. 23, $\mathrm{n}^{\circ}$ 3. pp. 294-310.

Heidegger, M. (1973 [1958], trad. fr.). « La question de la technique ». In Essais et conférences. Paris : Gallimard. pp. 9-48.

Herdina, P. \& Jessner, U. (2006). A dynamic Model of Multilingualism, Perspectives of Change in Psycholinguistics. Clevedon: Multilingual Matters.

Hofstadter, D. \& Sander, E. (2013). Surfaces and Essences: Analogy As the Fuel and Fire of Thinking. New York: Basic Books.

Hutchins, E. (1995). Cognition in the Wild. Cambridge, MA: MIT Press.

Jessner, U. (2006). Linguistic Awareness in Multilinguals. Edinburgh: Edinburgh University Press.

Kramsch, C. 2002. « Introduction. How can we tell the dancer from the dance? ». In Kramsch, C. (ed.) Language Acquisition and Language Socialization. Ecological Perspectives. pp. 1-30. London : Continuum.

Khreim, J.-M. (2008). Apport des technologies de l'information et de la communication (TIC) sur la dénativisation d'apprenants arabophones de l'anglais langue étrangère dans un contexte syrien. Thèse de doctorat : Université Sorbonne nouvelle-Paris 3.

Lahire, B. (1998). L'homme pluriel, les ressorts de l'action. Paris : Nathan

Laplane, D. (2001). La pensée sans langage. Études. 2001/3 - Tome 394. pp. 345-357.

Lave, J \& Wenger E. (1991). Situated Learning: Legitimate Peripheral Participation, Cambridge: CUP.

LeDoux, J. (2003). Neurobiologie de la personnalité, Paris : Odile Jacob.

Lenoir, Y. (2007). «L'habitus dans l'œuvre de Pierre Bourdieu : un concept central dans sa théorie de la pratique à prendre en compte pour analyser les pratiques d'enseignement ». Document du CRI et de la CRCIE. $\mathrm{N}^{\circ} 1$. Université de Sherbrooke.

Miras, G. (2014). Approche plurielle des liens musique parole pour la didactique de la prononciation du français en contexte de langue étrangère/seconde. Thèse en didactique des langues et des cultures Université Sorbonne Nouvelle - Paris 3. 
Narcy, J.-P. (1990). Apprendre une langue étrangère. Paris : Ophrys.

Narcy-Combes, J.-P. (2005). Didactique des langues et TIC - Pour une recherche-action responsable. Paris : Ophrys.

Narcy-Combes, J.-P. (2010). « L'illusion ontologique en didactique des langues », in Macaire D., Narcy-Combes, J.-P. \& Portine, H., (eds.) « Interrogations épistémologiques ». Le français dans le monde, Recherches et applications $n^{\circ} 48$. Paris : Clé International.

Narcy-Combes, J.-P. (2014). « De la complémentarité des domaines pour un didacticien des langues : quelle place pour les multilittéracies ». FLuL (Fremdsprachen Lehren und Lernen) 43 (2). pp. 29-42

Narcy-Combes, M.-F., Narcy-Combes, J.-P. \& Miras, G. (2015). « La didactique des langues à l'heure du numérique ». Langues, cultures et sociétés, vol. 1, No 2. pp. 1-20.

O'Connor, T., \& Wong, H. Y., « Emergent Properties », The Stanford Encyclopedia of Philosophy (Summer 2015 Edition), Edward N. Zalta (ed.), URL = http://plato.stanford.edu/archives/ sum2015/entries/properties-emergent/ consulté le 17 septembre 2016. Non paginé.

O'Grady, W. (2010). « Emergentism » in P. Hogan (dir.). The Cambridge Encyclopedia of the Language Sciences. Cambridge : Cambridge University Press. pp. 274-76.

Piaget, J. (1970) Psychologie et épistémologie : pour une théorie de la connaissance. Paris: GonthierDenoël.

Roehr-Braken, K. (2014). « Explicit Knowledge and Processes From a Usage-Based Perspective: The Developmental Trajectory of an Instructed L2 Learner ». Language Learning 64:4, December 2014. pp. 771-808

Simondon, G. (1989). L'individuation psychique et collective. Paris: Aubier.

Spear, G. E., \& Mocker, D. W. (1984). « The organizing circumstance: Environmental determinants in self-directed learning ». Adult education quarterly, 35(1). pp. 1-10.

Vygotski, L. S. (1997 [1934]). Pensée et langage. Paris : La dispute/SNEDIT.

Webster (en ligne) : https://www.merriam-webster.com/ consulté le 10 septembre 2016.

Williams, S. \& Hammarberg, B. (1998). «Language Switches in L3 Production : Implications for a Polyglot Speaking Model ». Applied Linguistics 19/3. pp. 295-333.

\section{NOTES}

1. Dans la mesure où cette contribution est une réponse à un article en anglais, son auteur pense que ses lecteurs accepteront des références en anglais.

2. http://plato.stanford.edu/entries/properties-emergent/ consulté le 17 septembre 2016.

3. Intervention de Véronique Castelloti.

4. Ce positionnement qui a été décrit et justifié dans d'autres publications (Narcy-Combes 2005, Narcy-Combes 2014, Narcy-Combes, M.-F. et al,. 2015, par ex.) est synthétisé ici par souci de clarification avec le risque de paraitre quelque peu sibyllin.

5. Parmi ces conditions notons (Miras, $2014: 11-13$ ) :

- La bipédie permettant une meilleure adaptation à l'environnement aurait favorisé des changements neurophysiologiques permettant un traitement accru d'unités linguistiques complexes et abstraites. 
- La Vie sociale : «pendant la période de Homo habilis et Homo rudolfensis, la taille des groupes de premiers hominidés avait tellement augmenté que les grognements n'étaient plus suffisant comme seul moyen d'expression d'engagement envers les autres membres du groupe ».

- L'augmentation de la taille du néocortex : l'acquisition du langage nécessite une capacité de mémorisation cognitive importante de par la nécessité de mémoriser un grand nombre d'énoncés formulaires (Legallois, $2009: 14$ ).

6. Voir le titre d'une communication de l'auteur à un symposium à Barcelone: Is LanguageS Education beyond the scope of applied linguistics ?et Narcy-Combes 2014.

\section{RÉSUMÉS}

Cet article est d'un type particulier, puisque son auteur répond à un auteur du même numéro qui retrace les débats d'une journée d'étude NEQ (Notion en questions), consacrée à l'émergentisme en janvier 2016. Le positionnement du répondant, didacticien de l'intervention, explique sa réaction. Une première partie situe donc l'auteur dans un courant transdisciplinaire où l'émergentisme a sa place dans une perspective plurilingue. La suite est autant une réaction à l'article de Marjolijn Verspoor qu'aux débats de la journée. L'apport des recherches présentées dans l'article initial est d'un grand intérêt, mais leurs limites confirment le besoin d'un travail transdisciplinaire incluant d'ailleurs la discipline des documents académiques.

This article has an unusual format as its author responds to another article in the same issue that relates the exchanges that took place during a Notion in Question (NEQ) seminar devoted to «Emergentism» in January 2016. The theoretical position of the respondent, a researcher in language learning and teaching, justifies his response. The first part of the article will describe this transdisciplinary position in which emergentism has a place in a plurilingual perspective. The second part is as much a response to Marjolijn's Verspoor (MV)'s contribution as to the debates on that day. The results of MV's research are rich and worthwhile but their very limitations justify the need for transdisciplinary collaboration in which the various fields of research complement each other.

\section{INDEX}

Mots-clés : transdisciplinarité, complémentarité, émergentisme, neurophysiologie, historicité Keywords : transdisciplinarity, complementarity, emergentism, neurophysiology, historicity

\section{AUTEUR}

\section{JEAN-PAUL NARCY-COMBES}

Jean-Paul Narcy-Combes est professeur émérite en sciences du langage au DILTEC (EA2288) à l'université Sorbonne Nouvelle - Paris 3. Il s'intéresse à l'épistémologie de la didactique des langues en questionnant l'impact et la place du numérique sur le développement langagier, par 
exemple.

Courriel : jean-paul.narcy-combes@wanadoo.fr 